Case Study

\title{
Lumbar extension traction alleviates symptoms and facilitates healing of disc herniation/ sequestration in 6-weeks, following failed treatment from three previous chiropractors: a $\mathrm{CBP}^{\circledR}$ case report with an 8 year follow-up
}

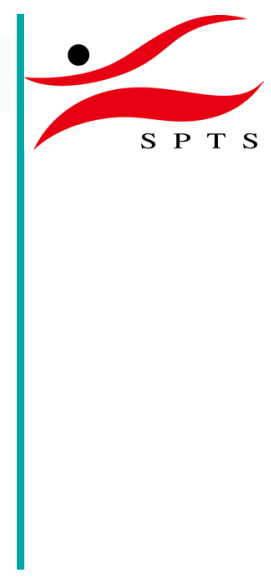

\author{
Paul A. OAKley ${ }^{1)^{*}}$, Deed E. Harrison ${ }^{2)}$ \\ 1) Private Practice: Newmarket, ON L3Y 8Y8, Canada \\ 2) $C B P$ NonProfit, Inc., USA
}

\begin{abstract}
Purpose] To present the outcome of a patient, having sciatica and MRI-verified disc herniation/sequestration who underwent Chiropractic BioPhysics ${ }^{\circledR}\left(\mathrm{CBP}^{\circledR}\right)$ protocol designed to improve the lumbar lordosis. [Subject and Methods] A 56-year-old male suffered from chronic low back pain and recent sciatica due to lumbar disc herniation despite being under continuous care from three previous chiropractors. Radiographic analysis revealed a lumbar hypolordosis and MRI confirmed disc herniation and sequestration at L4-L5. Generalized decreased lumbar range of motion and multiple positive orthopedic and neurologic tests were present. [Results] After 26 treatments of CBP lumbar extension traction over 9-weeks a total reduction of the disc herniation and sequestration occurred with concomitant improvement in neurologic symptoms. Continuing maintenance treatments, an 8 year follow-up shows no relapse of condition and patient remained in good health. [Conclusion] A patient with lumbar disc herniation/sequestration was successfully treated with CBP technique procedures including lumbar extension traction that achieved a significant healing of herniation and significant reduction in symptoms not obtained following traditional chiropractic procedures alone. The quick reduction in lumbar disc herniation would appear to be related to a segmental disc unloading force produced during extension traction procedures for increasing the lumbar curvature.

Key words: Lumbar lordosis, $\mathrm{CBP}^{\circledR}$, Disc herniation
\end{abstract}

(This article was submitted Jul. 17, 2017, and was accepted Aug. 23, 2017)

\section{INTRODUCTION}

The lumbar spine is the most common spinal location for disc herniation. Despite lumbar disc herniation (LDH) being one of the most frequent reasons for lumbar surgery ${ }^{1}$, there is still conflicting evidence regarding its suitability and long-term outcomes for many cases. Brox ${ }^{2}$, for example, reported "there is strong evidence that in carefully selected patients with sciatica due to lumbar disc prolapse, discectomy provides faster relief from the acute attack than conservative treatment." However, there is still no consensus as to whether the improved outcomes after discectomy are maintained longer than 6 months ${ }^{2)}$.

Therapists providing conservative care for LDH need to know which therapies provide effective outcomes in specific subgroups of patient populations ${ }^{3}$. Since common symptoms of LDH include back pain, leg pain, and sciatica, and as it is well known the intervertebral disc can be a source of direct and indirect pain, the chiropractor is undoubtedly presented with a large population of these patients ${ }^{4)}$.

*Corresponding author. Paul A. Oakley (E-mail: docoakley.icc@gmail.com)

(C2017 The Society of Physical Therapy Science. Published by IPEC Inc.

(c) (i) $\odot$ This is an open-access article distributed under the terms of the Creative Commons Attribution Non-Commercial No Derivatives

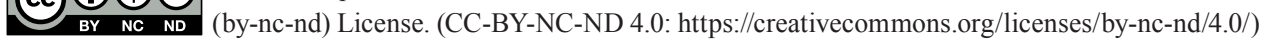


Table 1. 36-item short form survey values for initial (May, 2007), and first two follow-up assessments (Aug \& Dec, 2007)

\begin{tabular}{lcccccccc}
\hline & PF & Lim PH & Lim EP & E/F & EWB & SF & Pain & GH \\
\hline Norm & 71 & 53 & 66 & 52 & 70 & 79 & 71 & 57 \\
May 2007 & 40 & 0 & 100 & 35 & 64 & 37.5 & 22.5 & 65 \\
Aug 2007 & 55 & 0 & 100 & 60 & 80 & 62.5 & 55 & 70 \\
Dec 2007 & 75 & 100 & 100 & 70 & 92 & 100 & 77.5 & 75 \\
\hline
\end{tabular}

PF: Physical functioning; Lim PH: Role limitations due to physical health; Lim EP: Role limitations due to emotional problems; E/F: Energy/fatigue; EWB: Emotional well-being; SF: Social functioning; GH: General health

There is an expanding evidence base for spinal manipulative therapy (SMT) $)^{5)}$, as well as flexion-distraction manipulation $^{6-12)}$, and more recently lumbar extension traction (LET) $)^{13,14)}$ for patients suffering from LBP and radiculopathy. In one case using the LET method in combination with SMT led to a successful outcome in a patient with chronic LDH after the patient was unsuccessfully treated by $\mathrm{SMT}^{13}$ ). In a randomized clinical trial (RCT), LET with conventional treatment showed superior results to conventional treatment alone ${ }^{14)}$. Since lumbar kinematics, functional activity, and neurophysiological parameters were improved, the authors suggest that a normal lumbar lordotic curve is essential for normal neural function ${ }^{14)}$.

Due to the invasiveness and uncertainty of lumbar spine disc surgery, the growing evidence for alternative therapies ${ }^{5-14)}$, the challenge of treating patients with discogenic pain ${ }^{15)}$, and the lack of uniform treatment ${ }^{16}$, conservative chiropractic treatment options will continue to be, and need to be studied.

This case describes the successful management using Chiropractic BioPhysics ${ }^{\circledR}$ technique $\left(\mathrm{CBP}^{\circledR}\right)$, specifically LET methods of a patient having MRI-verified posterior lumbar disc herniation and sequestration at L4-L5 with chronic low back pain and radiculopathy with an 8 year follow-up. We also discuss the biomechanical effects of this treatment.

\section{SUBJECT AND METHODS}

On May 18, 2007, a 56-year-old male presented with low back and left leg pain. He had suffered from constant low back pain with varying pain intensity for a period of two years; he had developed recent sciatica over the last month despite being under continuous chiropractic care by three different practitioners.

Previous chiropractic care was virtually continuous between seeing three different chiropractors over approximately two years, details of treatments were not attainable. Due to lack of symptomatic relief from his condition, he was motivated to keep trying different chiropractors until he presented to the lead author's clinic. Concurrently, he was seeing his medical doctor who had prescribed him NSAIDs and Tylenol 3, as well as referred him to a neurologist for management of his condition.

At his initial examination, the patient presented with low back and left leg pain. The pain was rated on a numerical rating scale (NRS) as $8-9 / 10(0=$ no pain; $10=$ bed ridden $)$ without use of medications. Scoring of the Oswestry chronic low back pain disability index (ODI) revealed a $46 \%$ disability ${ }^{17}$. The 36 -item, quality of life questionnaire $(\mathrm{SF}-36)^{18)}$ revealed the patient had 7/8 health indices below normal (Table 1). All lumbar range of motion values were limited and guarded. The patient could not perform lumbar flexion or extension due to severe pain elicitation. Muscle strength testing revealed weakness of the left leg flexors (4/5); all others were normal. Positive orthopedic tests included left leg straight leg raise at $30^{\circ}$, Bechterew's test on the left, and left toe walk. All other orthopedic tests were normal. Posture evaluation ${ }^{19)}$ revealed the following: forward head translation $(+\mathrm{TzH})$, left thoracic translation $(+\mathrm{TxT})$, thoracic extension $(-\mathrm{RxT})$, right thoracic lateral bending $(+\mathrm{RzT})$, and anterior pelvis translation $(+\mathrm{TzP})$.

Radiographic mensuration procedures ${ }^{20-22)}$ demonstrated hypolordosis of the lumbar spine (L1-L5 $=35^{\circ}$; normal $=40^{\circ 23}$, 24): Fig. 1), slight posterior thoracic translation $(12 \mathrm{~mm})^{25)}$, normal cervical curve $\left(\mathrm{C} 2-\mathrm{C} 7=34^{\circ}\right)^{26-29)}$, slight forward head posture $(14 \mathrm{~mm})$, and a small right head translation $(7 \mathrm{~mm})^{30}$.

Shortly after beginning care, on June 13, 2007, the patient received an MRI of the low back (Fig. 2; Fig. 3). Being a resident of Ontario, Canada the socialized health plan often necessitates 'wait times' for many non-critical procedures, including MRI scans. For this reason the patient opted to fly to Buffalo, New York to pay 'out-of-pocket' for an MRI scan. The MRI report revealed the following: "A large inferiorly sequestered left posterolateral L4-5 disc herniation extending into the left $L 5$ lateral recess with severe compression of the left L5 and left S1 nerve roots; a small to moderate left posterolateral to left lateral foraminal L4-5 disc level herniation; and a bulging annulus fibrosus of L2-3, L3-4, and L5-S1."

The patient was put on CBP technique protocol of care utilizing mirror image ${ }^{\circledR}$ extension traction procedures $^{31-33)}$. During the first two treatments only, SMT, heat and ice on an inversion table were provided. Thereafter, these as well as CBP ${ }^{\circledR}$ 3-point bending LET 13, 14, 34-36) was provided (Fig. 4).

The patient was treated three times a week for six months with periodic re-examinations to document response to care and the need for continued care, if any. Thereafter, the patient was recommended a 'supportive care' plan and treated once per month. As of August 17, 2011 the patient had received a total of 114 treatments. A comprehensive examination and follow-up lateral lumbar radiographs were obtained at the 3-month, 6-month, 4.25, and an 8 year follow-up corresponding 


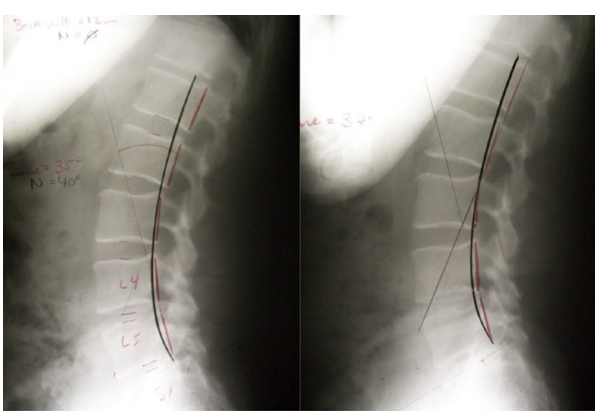

Fig. 1. Left: Pre lateral lumbar radiograph showing a slight loss of lordosis $\left(35^{\circ}\right)$. Right: Post lateral lumbar radiograph showing almost identical alignment $\left(37^{\circ}\right)$

Despite this small alignment improvement, the L4 disc had completely healed after 6-weeks of lumbar extension traction. The black line indicates normal alignment, the red line highlights the position of the patient's posterior vertebral body margins. The lordosis angle is measured as the angle between the L1 and L5 posterior body margins.

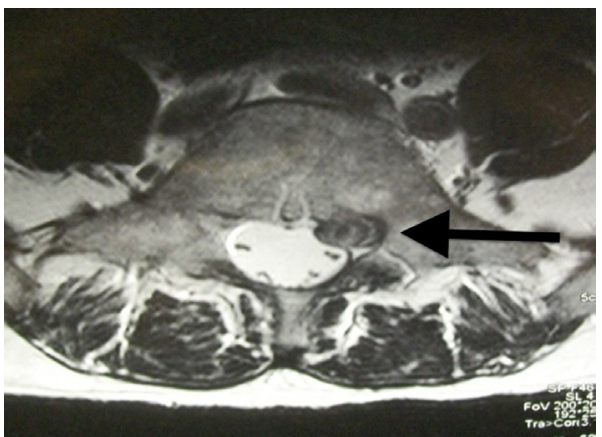

Fig. 3. MR image demonstrating an L4-L5 lumbar disc herniation/sequestration on a coronal slice

The large inferiorly sequestered left posterolateral L4-5 disc herniation extending into the left L5 lateral recess that resulted in compression of the left L5 and left S1 nerve roots.

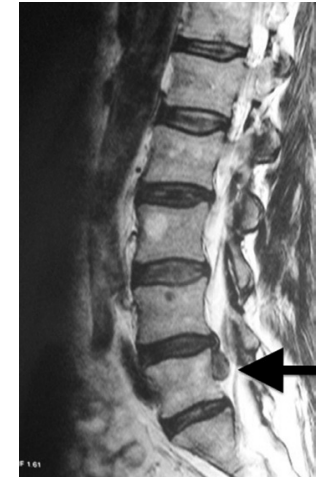

Fig. 2. MR image demonstrating an L4-L5 lumbar disc herniation/sequestration on a lateral slice

The bulging annulus fibrosus at L4-L5, and the inferiorly sequestered disc material.

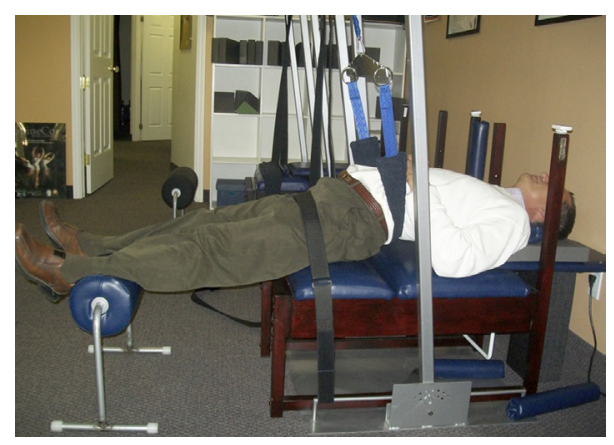

Fig. 4. Lumbar extension traction to increase the lumbar lordosis

to his 27th, 72nd, 114th, and 142nd treatment in accordance with evidence based protocols utilizing CBP technique LET procedures $^{13,14,34-36)}$. Examination results are reported from these four re-evaluation dates (Table 2).

Traction consisted of the patient supine on a Promote-Chiropractic-Supply traction table (Saugus, MA). A padded strap was placed under the mid-low lower back and hooked on to a spreader bar that is attached to a pulley system. The angle of pull of the lumbar traction was approximately 20 degrees towards the feet ${ }^{32}$. Traction duration began with five minutes on his third visit and progressed to a maximum of 15 minutes on his 7th visit to achieve appropriate ligamentous creep of the spine $^{37)}$. After the traction pull became significant, on the 11th visit, a small block was placed under the patient's torso to prevent posterior translation of the thorax in relation to the pelvis ${ }^{33,34)}$.

\section{RESULTS}

On August 2, 2007, the patient received a second MRI investigation of the lumbar spine. This was the advanced imaging that was originally requested from the neurologist approximately two months previous. The MR report indicated no evidence of lumbar herniation or sequestration; it stated: "The disc spaces are maintained. Bones, joints, and discs are within normal limits. The canal and lateral recesses are widely patent with no cause for pain detected."

On August 3, 2007 a comprehensive re-examination was performed corresponding with the patient's 27th treatment. The patient rated his low back as a $0-1 / 10$ on the NRS, the pain was limited to a central focal spot at approximately L4-S1. SF-36 revealed improvement in 6/8 health catagories (Table 1). Palpation revealed tight low back paraspinal muscles; described as 
Table 2. Values of selected measures for the initial, 6-week, 7-month, and 8 year follow-up assessments

\begin{tabular}{lcccc}
\hline \multicolumn{1}{c}{ Test } & Initial values & 6-weeks (27 txts) & 7-months (72 txts) & 8 -years (142 txts) \\
\hline NRS & $8-9 / 10$ & $1 / 10$ & $0-3 / 10$ & $0-3 / 10$ \\
ODI & $46 \%$ & $30 \%$ & $18 \%$ & $20 \%$ \\
ARA & $35^{\circ}$ & $37^{\circ}$ & $38^{\circ}$ & $39^{\circ}$ \\
MRI & Positive & Negative & N/A & N/A \\
Activity & None & Trying to run/golf & Normal & Normal \\
\hline
\end{tabular}

NRS: numerical rating scale ( $0=$ no pain; $10=$ worst pain ever); ODI: Oswestry low back pain disability questionnaire; ARA: absolute rotational angle $\left(40^{\circ}\right)$; MRI: magnetic resonance imaging; Activity: functional activity able to perform

tender due to playing golf a week previously. Patient's sciatic leg pain was dramatically improved with only occasional sharp pains on the left lateral proximal aspect of the calf. Range of motion of the low back was only limited slightly in rotation and flexion without pain, although tightness was felt during lateral bending. The ODI revealed a 30\% disability. Lumbar radiograph revealed a $37^{\circ}$ lordosis.

On December 21, 2007, a comprehensive re-examination was performed corresponding to the patient's $72 \mathrm{nd}$ treatment. The patient reported that his leg only occasionally bothered him, and rated his low back pain as 2-3/10 at a maximum after 'over doing it.' He reported he was working out heavily, including cross country running and playing golf. Lumbar range of motion was slightly reduced in all directions, albeit, with the exception of extension, without any pain. Palpation revealed only a focal pain sensation at L4-S1 and the left hip. The ODI indicated an 18\% disability. The SF-36 revealed improvement in $7 / 8$ health categories (Table 1). Lumbar curve measured $38^{\circ}$ on the lateral radiograph. Following the 7 -month exam, the patient was put on a supportive care schedule, consisting of treatments once per month.

On August 17, 2011 a comprehensive re-examination was performed corresponding to the patient's 114th treatment, 4 years and three months since his initial presentation. He stated his health was 'as good as it gets' and ran 30-35 miles/week and could golf as much as he liked without any back problems. He also stated that he recently drove from Florida to Toronto and in 15 hours of continuous driving and had no back pains afterward. He stated his low back was always slightly tender rating it at 1-2/10 (NRS). Lumbar range of motion was limited in flexion with no pain or discomfort. All other orthopedic tests were normal. Lumbar curve measured $39^{\circ}$ on the lateral radiograph, the ODI indicated a $22 \%$ disability. The SF-36 was not performed.

The patient continued a once per month treatment schedule. The final assessment included for this report was on May 27, 2015; the patient had received 142 treatments over 8 years (May 18, 2007-May 27, 2015). His low back health status has not changed for several years. He reports to be very well and that the only time his back bothers him is when he 'over does it,' such as when lifting things too heavy, he feels it the next day. This only may occur at most two times a month. He still runs 4-5 times a week a distance of 7-10 miles. All orthopedic tests were normal although he demonstrated a mild limitation in lumbar flexion and bilateral bending. Lumbar curve ARA measured $39^{\circ}$, ODI indicated a $20 \%$ disability; the SF-36 was not performed. The patient consented to the publication of these results.

\section{DISCUSSION}

This case demonstrates the quick resolution of an L4-L5 lumbar disc herniation and sequestration after beginning CBP lumbar extension traction methods in a patient who had a worsening of his back condition with a new onset of sciatica shortly prior to presentation, despite being under previous chiropractic treatment. Specifically, the disc lesion was resolved in 6-weeks verified by MRI, and this also corresponded with significant quality of life improvements as documented in health questionnaires (Tables 1 and 2).

Studies on the natural history of lumbar disc herniation have determined that this condition may be considered more benign than previously thought ${ }^{38)}$. This is because disc herniations have a tendency to resolve with time ${ }^{39}$. In fact, the majority of lumbar disc herniations tend to resolve with conservative treatment; that is, nonsurgically ${ }^{38)}$.

Vroomen et al. found that $73 \%$ of patients were found to have reasonable to major reduction in herniation after only 12 weeks ${ }^{40)}$. Cribb et al. found 14 of 15 patients having massive lumbar disc herniations demonstrated dramatic resolution of the herniation at a mean of 24 months following non-operative treatment ${ }^{38)}$. The timeline in this case was 6-weeks between positive and negative MR findings which is a shorter timeline than that determined by both Vroomen et al. ${ }^{40)}$ and Cribb et al. ${ }^{38)}$.

Chiropractors have apparently 'successfully' treated patients with lumbar disc herniation utilizing a plethora of therapies including SMT, paraspinal stimulation, physiotherapy, exercises, ice, heat, stretching, nutritional consultation, ergonomic consultation, Activator adjusting instrument, lumbosacral support, positive galvanism, distraction traction, flexion-distraction traction, and LET $^{5-14,41)}$. All of these methods have been used in a multimodal approach, i.e. not in isolation. The upside to this is that multimodal approaches have been found to be superior to single treatment approaches for chronic low back pain for example ${ }^{42)}$, however, if there is a very specific treatment that is ideal for patients with radiculopathy it can only be 
theoretically explained from the multimodal evidence that currently exists.

Despite clinical studies documenting successful outcomes in lumbar herniation patients, one must remember that the natural course of sciatica due to disc herniation is generally favorable but individually unpredictable ${ }^{43)}$. Specifically, some patients may only recover after prolonged conservative therapy ${ }^{42)}$. The case by Paulk and Harrison ${ }^{13)}$, for example, demonstrated the eventual successful outcome of a patient receiving extension lumbar traction who had chronic disc herniation. Similar to this study, the patient in the Paulk case also had unsuccessful, prior chiropractic SMT intervention ${ }^{13)}$.

Although there has been some evidence for SMT to benefit those with lumbar disc herniation ${ }^{5)}$, the first meta-analysis on the effectiveness of SMT for this condition concluded that it was neither more or less effective than other kinds of conservative care ${ }^{44)}$. Others have concluded that no conclusion can be drawn about various choice treatments, such as SMT, bed rest, medication, or physical therapy for patients suffering from LBP with radiculopathy ${ }^{45,46)}$.

In the only randomized clinical control trial of LET techniques on patients with discogenic lumbosacral radiculopathy ${ }^{14)}$, Moustafa et al. found clinically and statistically significant improvements in lumbar lordosis, Oswestry disability index, back and leg pain, Modified Schober test, latency and amplitude of H-reflex, and intervertebral movements. The control was treated with a 'conventional' treatment of hot packs and interferential therapy and the treatment group received the same plus LET. Both groups were treated at a frequency of three times per week for 10 weeks. The statistically improved values for the treatment group over the control was found after the 10 weeks of treatment as well as being maintained at a 6-month follow-up with no care. There were 32 patients in each group, matched for age, height, weight, gender, smoking, and use of medication for low back pain.

Since both groups in the Moustafa trial ${ }^{14)}$ received the same treatment (hot packs and interferential therapy), where the treatment group also received LET, the better functional and pain outcome measures were likely a result of the extension traction procedures. We propose that lumbar extension traction results in the biomechanical decompression of the lumbar spine aiding the body in the re-absorption and healing of the damaged discal material. There is accumulating evidence that lumbar extension is a biomechanical approach advantageous for treating lumbar disc herniation ${ }^{47)}$. Unlike other traction techniques, such as flexion-distraction or pure distraction, extension traction also repositions the lumbar spine into a more extended position $\left.{ }^{13}, 14,34-36\right)$, ie. a more lordotic, natural position for those presenting with hypolordosis.

Thus, the effects of the traction treatments may be lasting; that is, a patient who walks around with a closer to ideal lordosis will inadvertently exert less traumatic compression forces onto the damaged/healing disc while walking around; especially when flexing forwards (because started from a more extended position). This has been determined for the cervical spine by Takeshima et al ${ }^{48}$. Evaluating neutral as well as flexion and extension radiographs on different static neutral initial cervical spine alignments, they determined that those with cervical kyphosis could achieve less end-range lordosis angle than those with a normal lordotic spine, and vice versa, those with normal lordosis could achieve less end-range kyphosis than those with an initial kyphotic alignment. The static neutral alignment of the spine likely has a reciprocal influence on the dynamic stress-strain forces exerted onto the healing spinal discs through normal daily movement.

Much research is needed to elucidate the biomechanical effects of the application of treatment therapies onto the lumbar spine. Specifically, how the differing treatments influence the biomechanical characteristics of a herniated disc and how spinal extension may contribute positively to the healing process ${ }^{47}$.

The limitations of this case are as those expected from a single case report, a sample size of one. In addition, the subject received a treatment protocol that included chiropractic SMT, heat packs, ice, and inversion table, and not LET exclusively. Heat and ice therapy would not be expected to have any lasting effect on either increasing the lordosis or healing a herniation $^{49)}$. In regard to SMT, although there is evidence it is helpful for treating disc disease ${ }^{5)}$, it has not been shown to increase the lateral curves of the spine ${ }^{50,51)}$, and specifically the lumbar lordosis ${ }^{52)}$.

\section{REFERENCES}

1) Taylor VM, Deyo RA, Cherkin DC, et al.: Low back pain hospitalization. Recent United States trends and regional variations. Spine, 1994, 19: 1207-1212, discussion 13. [Medline] [CrossRef]

2) Brox JI: The contribution of RCTs to quality management and their feasibility in practice. Eur Spine J, 2009, 18: 279-293. [Medline] [CrossRef]

3) Gudavalli MR, Cambron JA, McGregor M, et al.: A randomized clinical trial and subgroup analysis to compare flexion-distraction with active exercise for chronic low back pain. Eur Spine J, 2006, 15: 1070-1082. [Medline] [CrossRef]

4) Troyanovich SJ, Harrison DD, Harrison DE: Low back pain and the lumbar intervertebral disk: clinical considerations for the doctor of chiropractic. J Manipulative Physiol Ther, 1999, 22: 96-104. [Medline] [CrossRef]

5) Leemann S, Peterson CK, Schmid C, et al.: Outcomes of acute and chronic patients with magnetic resonance imaging-confirmed symptomatic lumbar disc herniations receiving high-velocity, low-amplitude, spinal manipulative therapy: a prospective observational cohort study with one-year follow-up. J Manipulative Physiol Ther, 2014, 37: 155-163. [Medline] [CrossRef]

6) Cox JM: Lumbosacral disc protrusion: a case report. J Manipulative Physiol Ther, 1985, 8: 261-266. [Medline]

7) Neault CC: Conservative management of an L4-L5 left nuclear disk prolapse with a sequestrated segment. J Manipulative Physiol Ther, 1992, 15: 318-322. [Medline]

8) Cox JM, Hazen LJ, Mungovan M: Distraction manipulation reduction of an L5-S1 disk herniation. J Manipulative Physiol Ther, 1993, 16: 342-346. [Medline]

9) Hession EF, Donald GD: Treatment of multiple lumbar disk herniations in an adolescent athlete utilizing flexion distraction and rotational manipulation. J 
Manipulative Physiol Ther, 1993, 16: 185-192. [Medline]

10) BenEliyahu DJ: Magnetic resonance imaging and clinical follow-up: study of 27 patients receiving chiropractic care for cervical and lumbar disc herniations. J Manipulative Physiol Ther, 1996, 19: 597-606. [Medline]

11) Bergmann TF, Jongeward BV: Manipulative therapy in lower back pain with leg pain and neurological deficit. J Manipulative Physiol Ther, $1998,21: 288-294$. [Medline]

12) Morris CE: Chiropractic rehabilitation of a patient with S1 radiculopathy associated with a large lumbar disk herniation. J Manipulative Physiol Ther, 1999 , 22: 38-44. [Medline] [CrossRef]

13) Paulk GP, Harrison DE: Management of a chronic lumbar disk herniation with chiropractic biophysics methods after failed chiropractic manipulative intervention. J Manipulative Physiol Ther, 2004, 27: 579. [Medline] [CrossRef]

14) Moustafa IM, Diab AA: Extension traction treatment for patients with discogenic lumbosacral radiculopathy: a randomized controlled trial. Clin Rehabil, 2013, 27: 51-62. [Medline] [CrossRef]

15) Simon J, McAuliffe M, Shamim F, et al.: Discogenic low back pain. Phys Med Rehabil Clin N Am, 2014, 25: 305-317. [Medline] [CrossRef]

16) Malik KM, Cohen SP, Walega DR, et al.: Diagnostic criteria and treatment of discogenic pain: a systematic review of recent clinical literature. Spine J, 2013, 13: 1675-1689. [Medline] [CrossRef]

17) Hudson-Cook N, Tomes-Nicholson K, Breen A: A revised Oswestry disability questionnaire. In: Roland M, Jenner JR, eds. Back pain: new approaches to rehabilitation and education. Manchester: Manchester University Press, 1989, pp 187-204.

18) Ware JE Jr, Sherbourne CD: The MOS 36-item short-form health survey (SF-36). I. Conceptual framework and item selection. Med Care, 1992, 30: 473-483. [Medline] [CrossRef]

19) Harrison DD: Abnormal postural permutations calculated as rotations and translations from an ideal normal upright static spine. In: Sweere JJ, ed. Chiropractic Family Practice. Gaithersburg: Aspen Publishers, 1992, pp 1-22.

20) Harrison DE, Harrison DD, Cailliet R, et al.: Cobb method or Harrison posterior tangent method: which to choose for lateral cervical radiographic analysis. Spine, 2000, 25: 2072-2078. [Medline] [CrossRef]

21) Harrison DE, Harrison DD, Cailliet R, et al.: Radiographic analysis of lumbar lordosis: centroid, Cobb, TRALL, and Harrison posterior tangent methods. Spine, 2001, 26: E235-E242. [Medline] [CrossRef]

22) Harrison DE, Holland B, Harrison DD, et al.: Further reliability analysis of the Harrison radiographic line-drawing methods: crossed ICCs for lateral posterior tangents and modified Risser-Ferguson method on AP views. J Manipulative Physiol Ther, 2002, 25: 93-98. [Medline] [CrossRef]

23) Harrison DD, Cailliet R, Janik TJ, et al.: Elliptical modeling of the sagittal lumbar lordosis and segmental rotation angles as a method to discriminate between normal and low back pain subjects. J Spinal Disord, 1998, 11: 430-439. [Medline] [CrossRef]

24) Janik TJ, Harrison DD, Cailliet R, et al.: Can the sagittal lumbar curvature be closely approximated by an ellipse? J Orthop Res, 1998, 16: 766-770. [Medline] [CrossRef]

25) Harrison DE, Cailliet R, Harrison DD, et al.: How do anterior/posterior translations of the thoracic cage affect the sagittal lumbar spine, pelvic tilt, and thoracic kyphosis? Eur Spine J, 2002, 11: 287-293. [Medline] [CrossRef]

26) Harrison DD, Harrison DE, Janik TJ, et al.: Modeling of the sagittal cervical spine as a method to discriminate hypolordosis: results of elliptical and circular modeling in 72 asymptomatic subjects, 52 acute neck pain subjects, and 70 chronic neck pain subjects. Spine, 2004, 29: 2485-2492. [Medline] [CrossRef]

27) Harrison DD, Janik TJ, Troyanovich SJ, et al.: Comparisons of lordotic cervical spine curvatures to a theoretical ideal model of the static sagittal cervical spine. Spine, 1996, 21: 667-675. [Medline] [CrossRef]

28) Harrison DD, Janik TJ, Troyanovich SJ, et al.: Evaluation of the assumptions used to derive an ideal normal cervical spine model. J Manipulative Physiol Ther, 1997, 20: 246-256. [Medline]

29) Harrison DE, Harrison DD, Hass JW: Structural rehabilitation of the cervical spine. Harrison CBP ${ }^{\circledR}$ Seminars, 2002.

30) Harrison DE, Harrison DD, Cailliet R, et al.: Cervical coupling during lateral head translations creates an S-configuration. Clin Biomech (Bristol, Avon), 2000, 15: 436-440. [Medline] [CrossRef]

31) Harrison DD, Janik TJ, Harrison GR, et al.: Chiropractic biophysics technique: a linear algebra approach to posture in chiropractic. J Manipulative Physiol Ther, 1996, 19: 525-535. [Medline]

32) Oakley PA, Harrison DD, Harrison DE, et al.: Evidence-based protocol for structural rehabilitation of the spine and posture: review of clinical biomechanics of posture (CBP) publications. J Can Chiropr Assoc, 2005, 49: 270-296. [Medline]

33) Harrison DE, Betz JW, Harrison DD, et al.: CBP structural rehabilitation of the lumbar spine: Harrison Chiropractic Biophysics Seminars, 2007.

34) Harrison DE, Cailliet R, Harrison DD, et al.: Changes in sagittal lumbar configuration with a new method of extension traction: nonrandomized clinical controlled trial. Arch Phys Med Rehabil, 2002, 83: 1585-1591. [Medline] [CrossRef]

35) Diab AA, Moustafa IM: Lumbar lordosis rehabilitation for pain and lumbar segmental motion in chronic mechanical low back pain: a randomized trial. J Manipulative Physiol Ther, 2012, 35: 246-253. [Medline] [CrossRef]

36) Diab AA, Moustafa IM: The efficacy of lumbar extension traction for sagittal alignment in mechanical low back pain: a randomized trial. J Back Musculoskeletal Rehabil, 2013, 26: 213-220. [Medline] [CrossRef]

37) Oliver MJ, Twomey LT: Extension creep in the lumbar spine. Clin Biomech (Bristol, Avon), 1995, 10: 363-368. [Medline] [CrossRef]

38) Cribb GL, Jaffray DC, Cassar-Pullicino VN: Observations on the natural history of massive lumbar disc herniation. J Bone Joint Surg Br, $2007,89: 782-784$. [Medline] [CrossRef]

39) Komori H, Shinomiya K, Nakai O, et al.: The natural history of herniated nucleus pulposus with radiculopathy. Spine, 1996, 21: 225-229. [Medline] [CrossRef]

40) Vroomen PC, de Krom MC, Knottnerus JA: Predicting the outcome of sciatica at short-term follow-up. Br J Gen Pract, 2002, 52: 119-123. [Medline]

41) Polkinghorn BS, Colloca CJ: Treatment of symptomatic lumbar disc herniation using activator methods chiropractic technique. J Manipulative Physiol Ther, 1998, 21: 187-196. [Medline]

42) Bogduk N: Management of chronic low back pain. Med J Aust, 2004, 180: 79-83. [Medline]

43) Vroomen PC, Wilmink JT, de KM: Prognostic value of MRI findings in sciatica. Neuroradiology, 2002, 44: 59-63. [Medline] [CrossRef] 
44) Assendelft WJ, Morton SC, Yu EI, et al.: Spinal manipulative therapy for low back pain. A meta-analysis of effectiveness relative to other therapies. Ann Intern Med, 2003, 138: 871-881. [Medline] [CrossRef]

45) Lawrence DJ, Meeker W, Branson R, et al.: Chiropractic management of low back pain and low back-related leg complaints: a literature synthesis. J Manipulative Physiol Ther, 2008, 31: 659-674. [Medline] [CrossRef]

46) Luijsterburg PA, Verhagen AP, Ostelo RW, et al.: Effectiveness of conservative treatments for the lumbosacral radicular syndrome: a systematic review. Eur Spine J, 2007, 16: 881-899. [Medline] [CrossRef]

47) Scannell JP, McGill SM: Disc prolapse: evidence of reversal with repeated extension. Spine, 2009, 34: 344-350. [Medline] [CrossRef]

48) Takeshima T, Omokawa S, Takaoka T, et al.: Sagittal alignment of cervical flexion and extension: lateral radiographic analysis. Spine, 2002,27 : E348-E355. [Medline] [CrossRef]

49) French SD, Cameron M, Walker BF, et al.: Superficial heat or cold for low back pain. Cochrane Database Syst Rev, 2006, 1: CD004750. [Medline]

50) Hurwitz EL, Aker PD, Adams AH, et al.: Manipulation and mobilization of the cervical spine. A systematic review of the literature. Spine, 1996, 21: 1746-1759, discussion 1759-1760. [Medline] [CrossRef]

51) Harrison DE, Cailliet R, Harrison DD, et al.: A new 3-point bending traction method for restoring cervical lordosis and cervical manipulation: a nonrandomized clinical controlled trial. Arch Phys Med Rehabil, 2002, 83: 447-453. [Medline] [CrossRef]

52) Plaugher G, Cremata EE, Phillips RB: A retrospective consecutive case analysis of pretreatment and comparative static radiological parameters following chiropractic adjustments. J Manipulative Physiol Ther, 1990, 13: 498-506. [Medline] 